\title{
Immunological basis of septal fibrosis of the liver in Capillaria hepatica-infected rats
}

Q.T. Lemos,

I.F. Magalhães-Santos and Z.A. Andrade
Laboratório de Patologia Experimental, Centro de Pesquisa Gonçalo Moniz, Fundação Oswaldo Cruz, Salvador, BA, Brasil

\section{Correspondence \\ Z.A. Andrade \\ Rua Valdemar Falcão, 121 \\ 41295-001 Salvador, BA \\ Brasil \\ E-mail: zilton@cpqgm.fiocruz.br}

Research supported by PAPES III (FIOCRUZ) and PRONEX.

Received August 20, 2002

Accepted June 3, 2003

\section{Abstract}

Rats infected with the helminth Capillaria hepatica regularly develop septal fibrosis of the liver similar to that induced by repeated ip injections of pig serum. Fibrosis starts when the focal parasitic lesions begin to show signs of resorption, thus suggesting an immunologically mediated pathogenesis of this fibrosis. To explore this possibility, the development of $C$. hepatica-related hepatic fibrosis was observed in rats exposed to worm antigens from the first neonatal day onward. Wistar rats $(150 \mathrm{~g})$ were either injected ip with an extract of $C$. hepatica eggs (protein concentration: $1 \mathrm{mg} / \mathrm{ml}$ ) or received immature eggs by gavage from the first neonatal day until adult life and were then infected with 500 embryonated eggs. Changes were monitored on the basis of serum levels of anti-worm antibodies and hepatic histopathology. Rats submitted to immunological oral tolerance markedly suppressed $C$. hepatica-related serum antibodies and septal fibrosis of the liver when infected with the helminth later on. Tolerance trials with ip injections of worm antigens gave essentially negative results. The partial suppression of septal fibrosis of the liver after the induction of immunological tolerance to $C$. hepatica antigens in rats indicates an immunological basis for the fibrosis and emphasizes the importance of immunological factors in the pathogenesis of hepatic fibrosis.

\section{Introduction}

Recent in vitro studies have demonstrated that soluble mediators are released by stimulated Kupffer cells (1-4), including several cytokines, such as transforming growth factor- $\beta$ (4). Mediators induce Ito's stellate cells to proliferate, differentiate and secrete extracellular matrix components (5). It is to be expected that this hepatic cellular axis, composed of two nonparenchymal cell types located within and around the hepatic sinuso-
Key words

- Capillaria hepatica model

- Hepatic fibrosis

- Immunological tolerance ids, would play a decisive role in the pathogenesis of the types of hepatic fibrosis designated as perisinusoidal and septal fibrosis.

Variable degrees of hepatic fibrosis were obtained in rats submitted to repeated and prolonged injections of foreign proteins (68). A peculiar model emerged from these experimental trials carried out to induce fibrosis by immunological means when whole pig serum or its albumin fraction was used (9). In the pig serum model, fibrosis gradually and insidiously developed, without be- 
ing preceded by hepatocellular necrosis or chronic inflammation (10), later progressing to a morphologic picture of cirrhosis (11). Bhunchet et al. (12) demonstrated that pig serum acts as an antigen, with the immune response to it being the decisive factor contributing to the pathogenesis of this peculiar type of fibrosis, which therefore proved to rest on an immunological basis. These investigators made rats tolerant to pig serum albumin by inoculating them with this protein from the first neonatal day to adult life. Then, at the end of the tolerance induction period, the animals developed extremely low antibody levels and no evidence of hepatic fibrosis when submitted to a further period of repeated injections with pig serum albumin. This procedure resulted in high serum antibody levels and widespread hepatic septal fibrosis in nontolerant controls.

In 1993, Ferreira and Andrade (13) described the regular appearance of septal fibrosis of the liver similar to that observed in the pig serum model in rats infected with the helminth Capillaria hepatica. In this host, C. hepatica usually dies and disintegrates by about 20-30 days of infection, after deposition of a considerable number of eggs within the liver. Septal fibrosis was seen to start after the focal parasitic necroinflammatory lesions were showing signs of encapsulation and resorption. It was then speculated that the slow and continuous release of soluble products from the encapsulated parasitic lesions was stimulating cells of the KupfferIto axis to produce fibrosis. The morphological and pathogenetic similarities between the pig serum model and the $C$. hepatica model were evident, probably sharing an immunological basis. To further understand the pathogenesis of fibrosis associated with $C$. hepatica infection, a trial on antigenic tolerance along the same lines as those used for the pig serum model seemed to be appropriate.

C. hepatica is a nematode that parasitizes the liver of a wide variety of mammalian hosts, especially rodents. It presents world- wide distribution and may cause human infection (14). After being deposited, the eggs are metabolically active within the liver for a prolonged period of time, but remain immature. They need to reach the outer environment (after the death of the host) and, after a period of 28-30 days, under favorable conditions of moisture and temperature, become embryonated (15). Host ingestion of immature eggs results in a "spurious" infection. In this case, the immature eggs pass through the digestive tract and are eliminated in the stools. Ingestion of embryonated eggs results in the production of larvae that invade the intestine and reach the liver to form adult worms, thus causing a "true" infection.

\section{Material and Methods}

\section{Animals}

Young healthy Wistar rats of both sexes, weighing approximately $150 \mathrm{~g}$, maintained on a commercial balanced diet and water $a d$ libitum were used. When manipulating the animals during the neonatal period, special care was taken to determine that the mothers were not going to refuse suckling by their litters.

\section{Experimental groups}

Group I. Twenty animals intraperitoneally (ip) injected with a saline extract of immature $C$. hepatica eggs (from 0.1 to 0.5 $\mathrm{ml})$. Injections were started on the very first day after birth and continued twice a week for 30 weeks until adult life. After completion of the tolerance-inducing procedures the animals were submitted to a surgical liver biopsy. Under sterile conditions and ether anesthesia, the abdomen was opened and a small piece of the liver was ligated and removed. No complications were recorded, and all animals recovered from surgery. Two days later they received by gavage $500 \mathrm{em}-$ bryonated $C$. hepatica eggs and a new liver 
biopsy was obtained 40 days later. The animals were then sacrificed 20 days later, 60 days after infection.

Group II. Twenty animals receiving by gavage a suspension of immature $C$. hepatica eggs (500 up to 2500) from the first neonatal day on instead of the injections of soluble antigen preparations. All other procedures were exactly the same as for the previous group.

Group III. Control of C. hepatica "true" infection. Ten rats were ip injected with saline from the first neonatal day to adult life under the same conditions as the animals of group I. After the 30th injection, they underwent a liver biopsy and were submitted to infection with 500 embryonated eggs administered by gavage. The animals were submitted to a surgical liver biopsy 40 days after inoculation and sacrificed 20 days later (60 days after infection).

Group IV. Control of C. hepatica "spurious" infection. Five healthy adult rats received by gavage 2500 immature eggs twice a week for 3 weeks. The animals underwent a liver biopsy 40 days after the last inoculation and were sacrificed 20 days later.

\section{Capillaria hepatica eggs and antigens}

Immature eggs were recovered from infected rats around the 40th day of infection. The livers were removed, washed and cleaned of debris and homogenized in a blender at $1300 \mathrm{rpm}$ for $5 \mathrm{~min}$ in the presence of a small amount of distilled water.

The material was washed with distilled water, the sediment was decanted, several times, and finally centrifuged. The sediment, containing the eggs, was suspended in a 3:2 mixture of saline and Percoll (Sigma, St. Louis, MO, USA) and again washed in distilled water. The clean eggs were placed on a Petri dish, humidified with $0.5 \%$ formalin and kept at room temperature (around $26^{\circ} \mathrm{C}$ ) for 28-30 days to embryonate. For antigen preparation, clean immature eggs were sus- pended in sterilized PBS and smashed in a manual homogenizer after several cycles of freezing and thawing. The supernatant was collected after centrifugation at 14,000 rpm for $40 \mathrm{~min}$ in a refrigerated Eppendorf centrifuge at $4^{\circ} \mathrm{C}$. Protein concentration was measured with a BCA protein assay reagent kit (Pierce, Rockford, IL, USA).

\section{Antibody detection}

Sera from the animals of the various groups were collected a) on the last day of the tolerance-inducing procedure, b) 40 days after challenge with a "true" or "spurious" infection with $C$. hepatica, and c) at the time of sacrifice. Sera were submitted to an ELISA test for detection of total immunoglobulin antibodies using a peroxidase-conjugated goat anti-rat IgG (Sigma). The plates were sensitized with $10 \mu \mathrm{g} / \mathrm{ml} C$. hepatica egg antigen diluted in carbonate buffer, $\mathrm{pH}$ 9.6, and readings were taken with a microplate reader (Thermomax spectrophotometer, Molecular Devices, Sunnyvale, CA, USA) at $450 \mathrm{~nm}$ connected to a computer with MDS-Soft Max.

\section{Histopathology}

Liver fragments obtained from surgical biopsies or at the time of sacrifice were immediately placed in buffered neutral formalin for fixation. The tissue was dehydrated in graded alcohol, cleared in xylene and embedded in paraffin. Five micrometerthick sections were stained with hematoxylin and eosin and Sirius-red for collagen visualization.

\section{Statistical analysis}

The Kruskal-Wallis and the Dunn multiple comparison nonparametric tests were used to compare serological data obtained from different experimental groups, considering $\mathrm{P}<0.05$ as significant. 


\section{Results}

Animals from group I, which were injected with egg antigen by the ip route, already exhibited high levels of serum anti-
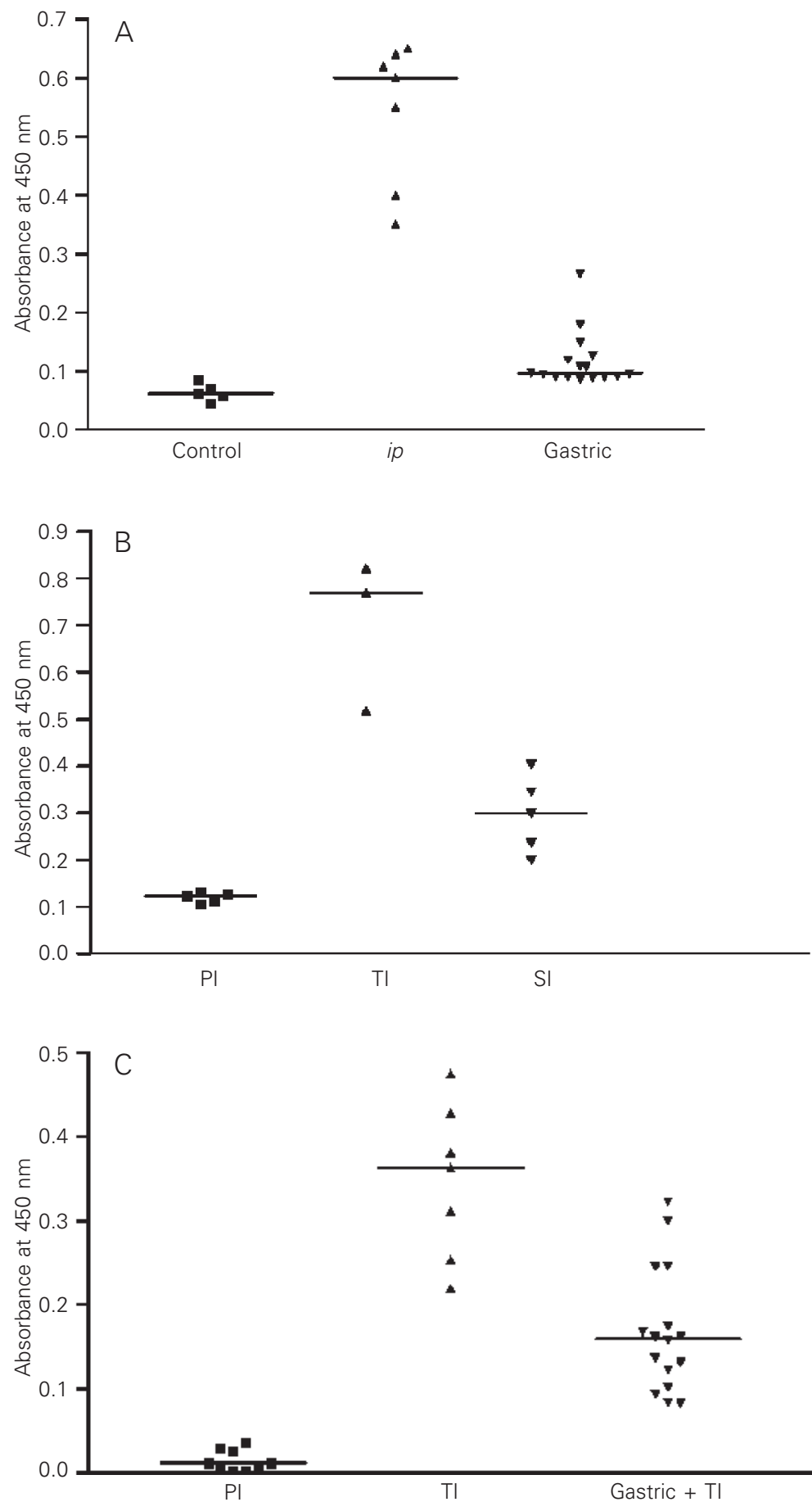

C. hepatica antibodies by the end of the tolerance induction period (Figure 1A). The concentration of these antibodies was increased 30 and 40 days after infection with embryonated eggs. Hepatic histopathologic examination 40 days after infection with embryonated eggs revealed encapsulated foci of chronic inflammation and necrosis containing dead worm debris and immature eggs. These results were similar to those obtained for group III, which was the control of "true" $C$. hepatica infection. In addition, throughout the hepatic parenchyma, fine fibrous septa were seen connecting portal spaces, central canals, portal spaces to central canals or vice-versa, in several combinations that resulted in a mosaic pattern (Figure $2 \mathrm{~A}$ and B).

Animals of group II, rendered tolerant by oral administration of immature $C$. hepatica eggs, exhibited either low titers or no $C$. hepatica antibodies after the tolerance in-

Figure 1. A, Levels of serum anti-Capillaria hepatica antibody (ELISA) in rats submitted to trials of immunological tolerance. Control, normal rat serum; ip (group I), animals repeatedly injected with egg antigen by the ip route since the neonatal period; gastric (group II), animals receiving by gavage immature $C$. hepatica eggs since the neonatal period. $P<0.001$ for control vs ip; $\mathrm{P}<0.05$ for control vs gastric; $\mathrm{P}<0.01$ for ip vs gastric (Kruskal-Wallis and the Dunn multiple comparison nonparametric tests). B, Anti-C. hepatica antibody levels (ELISA) in the sera of rats 40 days after oral administration of either immature ("spurious" infection) or embryonated ("true" infection) of $C$. hepatica eggs. PI, pre-infection sera; TI (group III), "true" infection (embryonated eggs); SI (group IV), "spurius" infection (immature eggs). $\mathrm{P}<0.01$ for $\mathrm{PI} v s \mathrm{TI} ; \mathrm{P}>0.05$ for $\mathrm{PI}$ vs SI (Kruskal-Wallis and the Dunn multiple comparison nonparametric tests). C, Anti-C. hepatica antibodies in the sera of rats submitted to a tolerance test by gastric administration of immature $C$. hepatica eggs, as compared to pre-infection levels and to "true" infection in previously normal rats. $\mathrm{Pl}$, pre-infection sera (normal rats); TI (group III), "true" infection; gastric + TI (group II), animals made tolerant by gastric administration of immature eggs and challenged with a "true" infection. $\mathrm{P}<0.001$ for $\mathrm{PI}$ vs Tl; $\mathrm{P}<0.01$ for $\mathrm{PI}$ vs gastric $+\mathrm{Tl} ; \mathrm{P}>0.05$ for TI vs gastric $+\mathrm{Tl}$ (KruskalWallis and the Dunn multiple comparison nonparametric tests). 
duction period (Figure 1A). Antibodies were present in all animals from this group after they were inoculated with embryonated eggs, but still at low levels (Figure 1C). Forty days after a true infection with $C$. hepatica, histopathologic alterations in the liver markedly differed from those seen in group I and in controls (group III). The focal parasitic lesions were present in the liver of tolerant animals, with encapsulation, necrosis and inflammation, but the eggs in the lesions seemed to be much less numerous than in controls, usually exhibiting a thin and transparent shell (Figure 2C). Septal fibrosis was limited to a few spurs radiating from the capsule of the focal parasitic lesions (Figure $3 \mathrm{~A}$ ), while the rest of the liver parenchyma maintained its normal structure. Occasionally, septal fibrosis extended further into small areas of the hepatic parenchyma, away from the parasitic lesions. In this case, the septa were fewer and thinner, sometimes incomplete when compared to controls. Some of the $C$. hepatica lesions still contained live worms surrounded by a scanty inflammatory reaction and no eggs, even by 60 days after inoculation with embryonated eggs (Figure 3B). Live worms are usually not found after the 40th day of infection in rats. Animals solely receiving immature eggs by gavage did not develop infection, but did present low titers of anti-C. hepatica antibodies in serum (Figure 1B). Microscopic examination of hepatic tissue did not show abnormalities in this group of animals.

The statistical analysis of the serological results is presented in Figure 1.

Although the median values did show

Figure 2. $A$ and $B$, Aspect of the septal fibrosis observed in the liver of rats infected with the helminth Capillaria hepatica (group III - "true" infection), 40 days after infection. Foci of egg deposition can be seen at the top of panel B. Sirius-red method for collagen, 200X. C, Encapsulated necroinflammatory lesion formed around dead worm debris and a few transparent eggs. Hematoxylin and eosin, 200X.
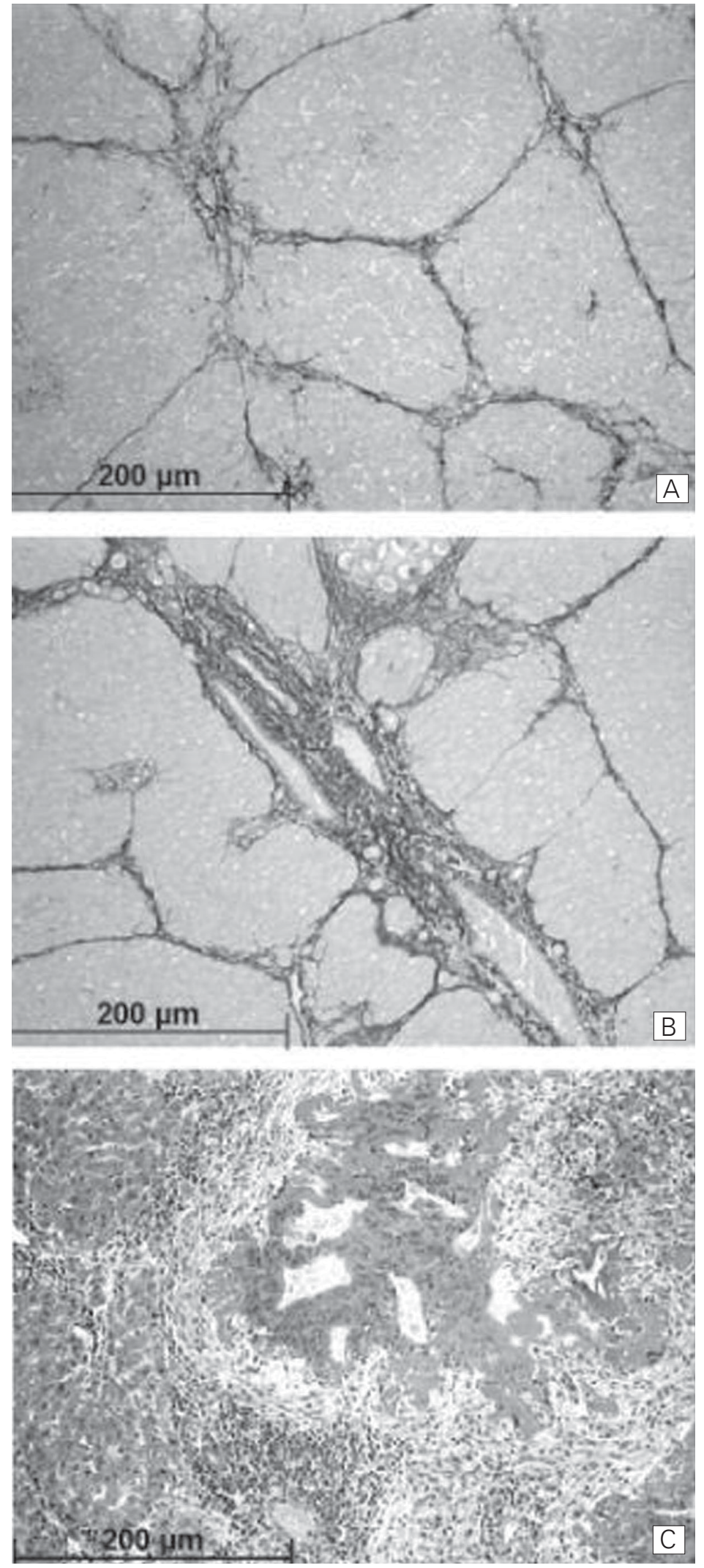
differences between the two experimental groups, these differences were not statistically significant, indicating only a partial state of immunosuppression.
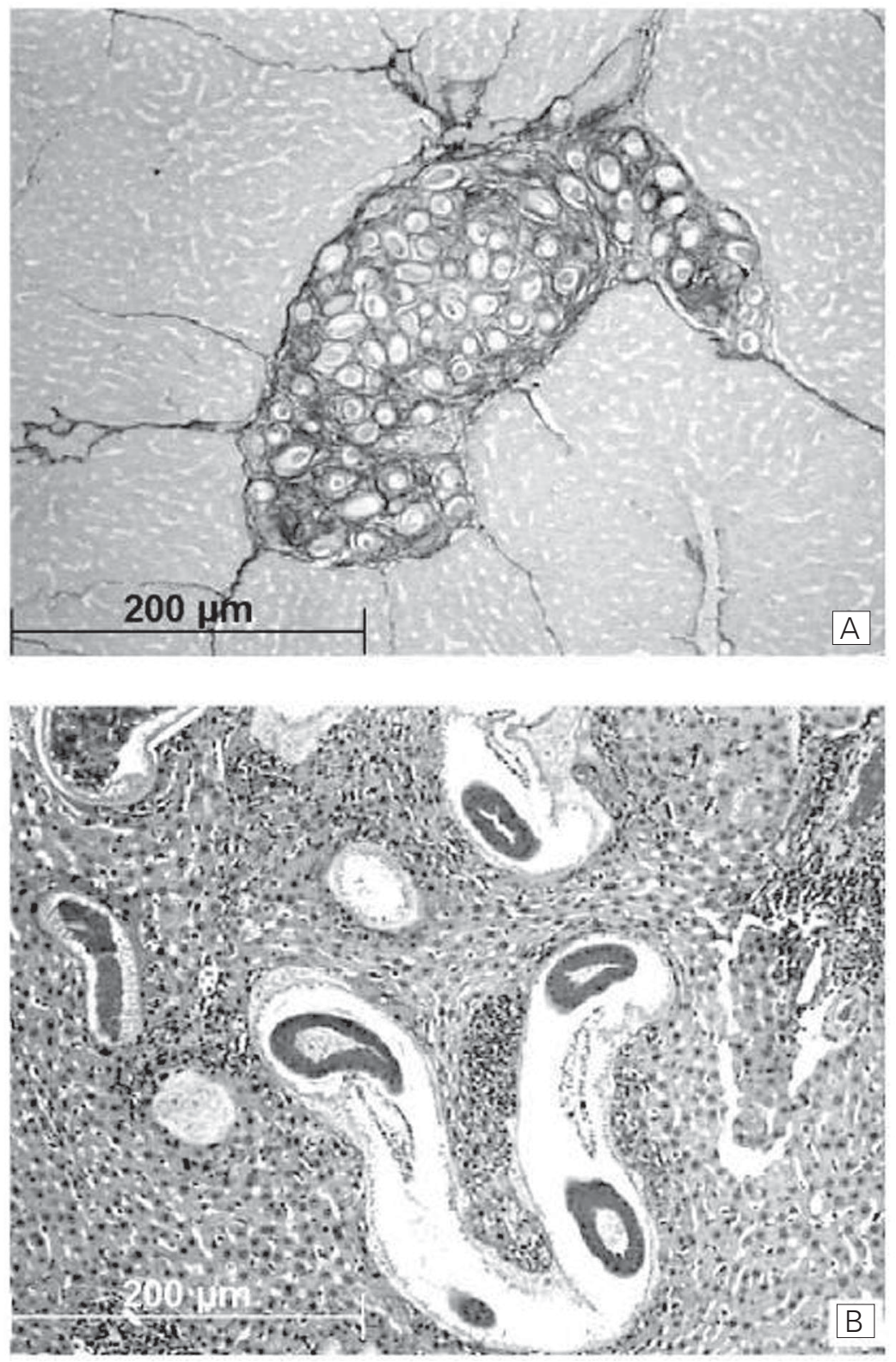

Figure 3. A, Large collection of immature Capillaria hepatica eggs in the liver of a rat made immunologically tolerant by oral administration of immature egg antigens from the first neonatal day until adult life (group II). Only a scanty amount of septal fibrosis radiates from the capsule of the parasitic lesion 40 days after a "true" infection. Sirius-red method for collagen, 200X. B, Young adult worms surrounded by a mild inflammatory reaction are present in the liver of a $C$. hepatica-tolerant rat from group II, 60 days after infection with embryonated eggs. Hematoxylin and eosin, 200X.

\section{Discussion}

The results obtained from the present investigation suggested that the task of making rats completely tolerant to $C$. hepatica is more difficult than making rats tolerant to whole pig serum or to pure pig serum albumin. This could be expected, since in the case of $C$. hepatica much more complex antigens are probable involved. Serum albumin is a single protein molecule, although it presents multiple antigenic determinants. However, in regard to C. hepatica, not only protein and somatic and metabolic polysaccharide antigens derived from the worms and their eggs, but also possibly neo-antigens derived from host-parasite interactions, can be expected to play a rather complex role in the process. The considerable reduction of septal fibrosis of the liver, which was observed in the animals receiving immature eggs by gavage from the neonatal period onward, can be interpreted as immunological tolerance, albeit partial. It can thus be assumed that septal fibrosis occurring in rat capillariasis has an immunological basis. Although tolerance has not been achieved by the parenteral administration of antigen, as was the case with pig serum-treated rats, as demonstrated by Bhunchet et al. (12), the similarity of the two models can also be assumed by their responses regarding the process of induced tolerance.

The behavior of serum antibodies is also in keeping with this conclusion. The upsurges of antibodies after the "tolerant" rats became infected with $C$. hepatica may indicate a derepression phenomenon. Higgins and Weiner (16) have called attention to the fact that disease manifestations can be partially or completely suppressed in tolerant hosts in a dose-dependent manner.

The use of ip injections of soluble antigens to make rats tolerant to $C$. hepatica was attempted because of the excellent results obtained by Bhunchet et al. (12) in their studies with the pig serum model. As men- 
tioned above, the ip route, under the present experimental circumstances, proved to be totally inadequate for the rat $C$. hepatica model. It is well known that not only the doses but also the route of administration of antigens have considerable effects on the immune response (17-20). Also, for the process of immunological tolerance it is important that the antigens remain for a prolonged period of time within the organism, otherwise only a transient tolerance would be induced (21). To emphasize the importance of the route of administration, Andrade and Godoy (22) demonstrated that rats treated with whole pig serum by subcutaneous injections failed to develop hepatic fibrosis. Under the same experimental protocol, septal hepatic fibrosis developed in $65 \%$ of the rats injected ip.

In attempts to induce hepatic fibrosis by immunological means, the $C$. hepatica model can be considered an alternative to the pig serum model. Although immunologically more complex, the $C$. hepatica model has the advantage of coursing with almost no mortality and with fibrosis occurring in $100 \%$ of the infected animals (13).

\section{References}

1. Friedman SL (2000). Molecular regulation of hepatic fibrosis, an integrated cellular response to tissue injury. Journal of Biological Chemistry, 275: 2247-2250.

2. Friedman SL \& Arthur JP (1989). Activation of cultured rat hepatic lipocytes by Kupffer cell conditioned medium. Journal of Clinical Investigation, 84: 1780-1785.

3. Shiratori Y, Greets A, Ichida T, Kawase T \& Wisse E (1986). Kupffer cells from $\mathrm{CCl}_{4}$-induced fibrotic livers stimulate proliferation of fatstoring cells. Journal of Hepatology, 3: 294-303.

4. Pinzani M \& Marra F (2001). Cytokine receptors and signaling in hepatic stellate cells. Seminars in Liver Disease, 21: 397-416.

5. Friedman SL, Roll FJ, Boyles J \& Bissel DM (1985). Hepatic lipocytes: the principal collagen-producing cells of normal rat liver. Proceedings of the National Academy of Sciences, USA, 82: 86818685.

6. Longcope WT (1913). Cirrhosis of the liver produced by chronic protein intoxication. Transactions of the Association of American Physicians, 28: 497-512.

7. Blackwell JB (1965). Cirrhosis resulting from repeated injections of antigens. Journal of Pathology and Bacteriology, 90: 245-258.

8. Fennel $\mathrm{RH}$ (1965). Chronic liver disease induced in rats by repeated anaphylactic shock. American Journal of Pathology, 47: 173-182.

9. Paronetto F \& Popper H (1966). Chronic liver injury induced by immunologic reactions. Cirrhosis following immunization with heterologous sera. American Journal of Pathology, 40: 1087-1101.

10. Rubin E, Hutterer F \& Popper H (1968). Experimental hepatic fibrosis without hepatocellular regeneration. A kinetic study. American Journal of Pathology, 52: 111-119.

11. Andrade ZA (1991). Contribution to the study of septal fibrosis of the liver. International Journal of Experimental Pathology, 72: 553562.

12. Bhunchet E, Eishi $Y$ \& Wake K (1996). Contribution of immune response to the hepatic fibrosis induced by porcine serum. Hepatol- ogy, 23: 811-817

13. Ferreira LA \& Andrade ZA (1993). Capillaria hepatica: a cause of septal fibrosis of the liver. Memórias do Instituto Oswaldo Cruz, 88: 441-447.

14. McQuown AL (1954). Capillaria hepatica. American Journal of Clinical Pathology, 24: 448-452.

15. Wright WH (1961). Observation on the life cycle of Capillaria hepatica (Bancroft, 1893) with a description of the adult. Canadian Journal of Zoology, 39: 167-182.

16. Higgins PJ \& Weiner HL (1988). Suppression of experimental autoimmune encephalomyelitis by oral administration of myelin basic protein and its fragments. Journal of Immunology, 140: 440-445.

17. Goto M, Yoshioka T, Young RI, Battelino T, Anderson CL \& Zeller WP (1997). A sublethal dose of LPS to pregnant rats induces TNFalpha tolerance in their 0-day-old offspring. American Journal of Physiology, 273: R1158-R1162.

18. Ilan Y, Prakash R, Davidson A, Jona V, Droguett G, Horwitz MS, Chowdhury NR \& Chowdhury JR (1997). Oral tolerization to adenoviral antigens permits long-term gene expression using recombinant adenoviral vectors. Journal of Clinical Investigation, 99: 10981106

19. Stiltz $L$ (2001). Induction of antigen specific tolerance by cyclosporin A. European Journal of Immunology, 22: 1995-2001.

20. Weigle WO (1991). Self-non self recognition by $T$ and B lymphocytes and their role in auto-immune phenomena. Arthritis and Rheumatism, 24: 1044-1053.

21. Smith KM, Eaton AD, Finlayson LM \& Garside P (2000). Oral tolerance. American Journal of Respiratory and Critical Care Medicine, 162: $175-178$

22. Andrade ZA \& Godoy A (1996). Influence of the route of administration of pig serum in the induction of hepatic septal fibrosis in rats. Memórias do Instituto Oswaldo Cruz, 91: 769 